\title{
Normal distribution of collagen IV in renal basement membranes in Epstein's syndrome
}

Ichiro Naito, Shinsuke Nomura, Satoko Inoue, Megumi Kagawa, Shinichiro Kawai, Yoshimi Gunshin, Kensuke Joh, Chizuko Tsukidate, Yoshikazu Sado, Gengo Osawa

\author{
Divisions of \\ Ultrastructural \\ Biology and \\ Immunology, Shigei \\ Medical Research \\ Institute, Okayama, \\ Japan \\ I Naito \\ $S$ Inoue \\ M Kagawa \\ Y Sado
}

Nephrology Division,

Department of

Medicine, Kawasaki

Medical School,

Kurashiki, Japan

S Nomura

G Osawa

\section{Division of Internal}

Medicine, Shigei

Hospital, Kurashiki,

Japan

S Kawai

\section{Department of}

Pediatrics, Faculty of Medicine, University

of Tokyo, Tokyo, Japan

Y Gunshin

Department of

Pathology II, The Jikei

University School of

Medicine, Tokyo, Japan

K Joh

Division of Pediatrics, Japanese Red Cross

Nagoya First Hospital,

Nagoya, Japan

C Tsukidate

Correspondence to: Dr Naito, Division of

Ultrastructural Biology,

Shigei Medical Research

Institute, 2117 Yamada,

Okayama, 701-02 Japan.

Accepted for publication 15 July 1997

\begin{abstract}
Background-Epstein's syndrome is defined as a subtype of Alport's syndrome, and is distinguished from the other subtypes by accompanying macrothrombocytopenia. Mutations in collagen IV genes are known to be involved in the pathogenesis of typical Alport's syndrome. However, the presence of an underlying genetic defect has not been demonstrated in Epstein's syndrome.

Aim-To clarify the involvement of collagen IV in Epstein's syndrome.

Methods-The distribution of the $\alpha(I V)$ chain was studied in renal specimens obtained from three patients with Epstein's syndrome using chain specific monoclonal antibodies and an antigen retrieval procedure.

Results-The patients showed a normal distribution of $\alpha(I V)$ chains: $\alpha 1$ (IV) and a2(IV) were expressed ubiquitously, whereas expression of $\alpha 3$ (IV) through to $\alpha 6$ (IV) chains was limited to the glomerular basement membrane, Bowman's capsular basement membrane, and/or a portion of the tubular basement membrane.

Conclusions-These results suggest that genes other than those encoding $\alpha($ IV) chains are responsible for the pathogenesis of Epstein's syndrome.

(F Clin Pathol 1997;50:919-922)
\end{abstract}

Keywords: Alport's syndrome; Epstein's syndrome; macrothrombocytopenia; collagen IV; deafness

Alport's syndrome is a progressive hereditary renal disease accompanying multiple abnormalities including sensorineural deafness, ocular abnormalities, macrothrombocytopenia, and diffuse leiomyomatosis. ${ }^{1}$ These abnormalities vary in expression and severity among families and patients. ${ }^{1}$ Epstein $e t a l^{2}$ described two unrelated families affected with hereditary nephritis, deafness, and macrothrombocytopenia (Epstein's syndrome). This is distinguished from other subtypes of Alport's syndrome by the presence of accompanying macrothrombocytopenia. Mutations in collagen IV genes are involved in the pathogenesis of X linked dominant ${ }^{3-5}$ and autosomal recessive Alport's syndrome, ${ }^{67}$ and abnormalities in the $\alpha$ (IV) chain distribution have been demonstrated using immunohistochemical methods. ${ }^{8-10}$ Previously, we have studied all 51 exons of the COL4A5 gene in two cases of
Epstein's syndrome, but no mutations were found. ${ }^{4}$ This result does not belie the possibility that mutations in the COL4A5 gene might account for the pathogenesis of Epstein's syndrome, because even in the $\mathrm{X}$ linked Alport's syndrome, mutations in the COL4A5 gene have been demonstrated in fewer than $50 \%$ of patients. $^{45}$ Epstein's syndrome is known to be an autosomal dominant disorder, ${ }^{2}$ which suggests the involvement of COL4A 3 or COL4A4, rather than COL4A5 in the pathogenesis of the disease.

Six different $\alpha($ IV) chains have been identified genetically and immunohistochemically. We prepared monoclonal antibodies specific for the human $\alpha 1$ (IV) through to $\alpha 6$ (IV) chains and used them for the immunohistochemical analysis of fresh frozen sections of human tissues. ${ }^{11}$ Antigen retrieval has extended their application to paraffin embedded sections. ${ }^{12} \mathrm{~A}$ combination of monoclonal antibodies and antigen retrieval has enabled a retrospective study of the $\alpha(\mathrm{IV})$ chain in rare diseases. To clarify the involvement of collagen IV in the pathogenesis of Epstein's syndrome, the distribution of all six $\alpha(\mathrm{IV})$ chains was determined in renal specimens obtained from Epstein's syndrome patients using these immunohistochemical procedures.

\section{Methods}

PATIENTS

Renal specimens were obtained from three patients from different families. The diagnosis of Epstein's syndrome was based on the presence of a combination of nephritis, deafness, and macrothrombocytopenia. However, none of the patients presented ocular abnormalities.

\section{Patient 1}

At 3 years old patient 1 had been diagnosed with idiopathic thrombocytopenic purpura (ITP). Sensorineural hearing loss was recognised at 6 years of age. Giant platelets were found on her peripheral blood smear. The patient was treated for ITP but her platelet count remained in the range of $1-2 \times 10^{3} / \mathrm{ml}$. Subsequently, the patient had proteinuria and microhaematuria. At the age of 16 , she underwent a renal biopsy. Light microscopy revealed an almost normal glomerular shape and mild fibrosis in the interstitium, which contained foam cells. Electron microscopy revealed reticulation of the lamina densa in the glomerular basement membrane (fig 1). 


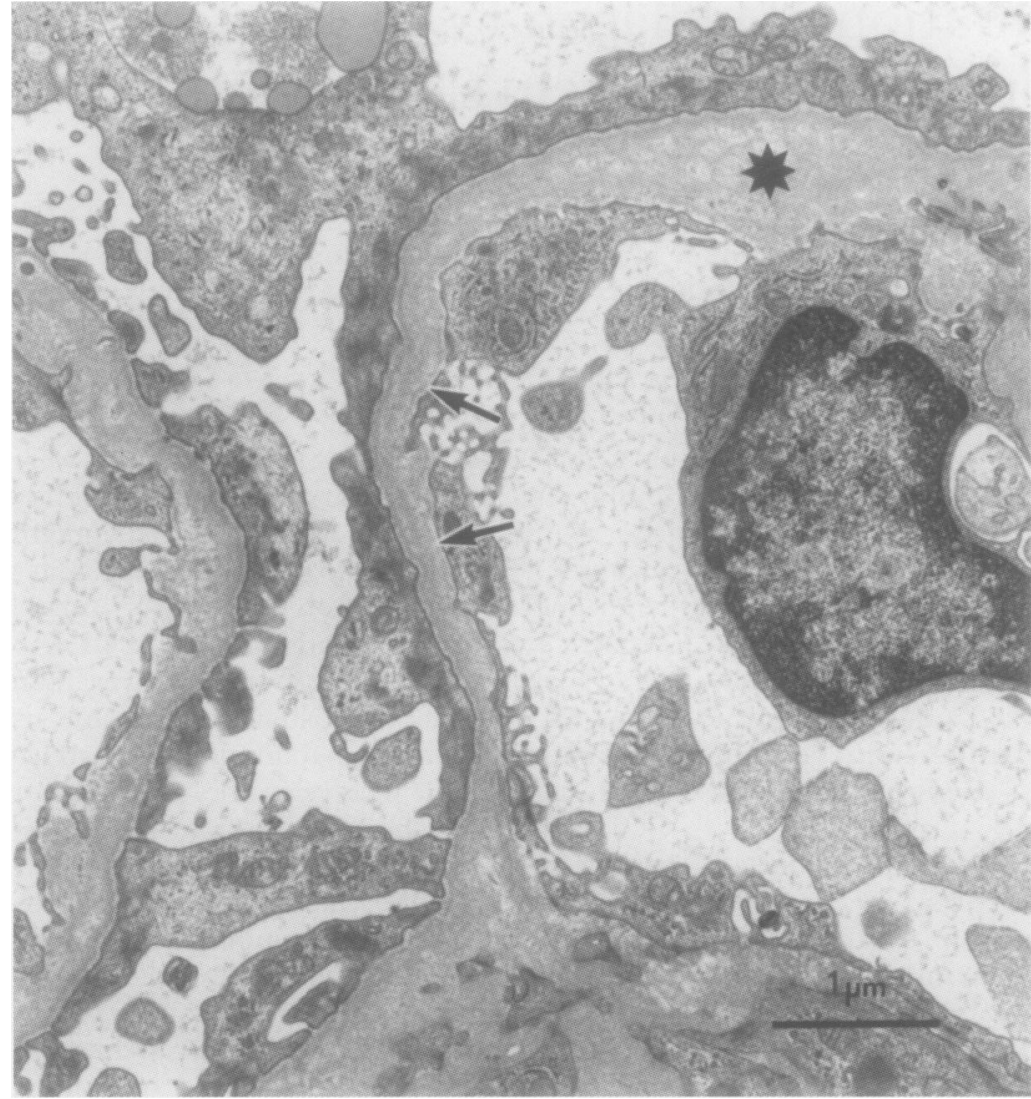

Figure 1 Electron micrograph of the glomerular basement membrane of patient 1. Reticulation (asterisk) and lamination (arrows) of the lamina densa are observed in the thickening glomerular basement membrane.

\section{Patient 2}

Since 4 years of age, patient 2 had been treated for ITP, but her platelet count did not recover. Proteinuria was detected at 8 years of age and then increased to $2 \mathrm{~g}$ /day. She was found to have thrombocytopenia $\left(4 \times 10^{3}-1 \times 10^{4} / \mathrm{ml}\right)$ with large platelets. She showed moderate sensorineural deafness but renal function was still in the normal range. A renal biopsy was performed at the age of 12 . Mild mesangial proliferative glomerulonephritis, interstitial fibrosis, and tubular atrophy were found. Electron microscopy revealed irregularity of the glomerular basement membrane and the electron dense granules in the partially split lamina densa of the glomerular basement membrane.

\section{Patient 3}

Patient 3 had a history of recurrent epistaxis from the age of 1 year. A bone marrow biopsy was performed at 3 years of age and he was diagnosed as suffering from ITP. He showed proteinuria of $4+$ and mild sensorineural hearing loss when 15 years old. One year later, he underwent a renal biopsy. Light microscopy revealed focal segmental sclerosis of the glomerulus and the expansion of the interstitium, which was infiltrated with lymphocytes and foam cells. Electron microscopy revealed thinning of the glomerular basement membrane. Owing to renal dysfunction, he was started on haemodialysis at 17 years of age. At the time of writing his platelet count was $1.7 \times 10^{4} / \mathrm{ml}$.
All three patients satisfied three or four of the 10 criteria for the diagnosis of Alport's syndrome proposed by Gregory et al. ${ }^{1}$ No history of hearing abnormality, renal abnormality, or macrothrombocytopenia existed in the families and relatives of these three patients. All 51 exons of the COL4A 5 gene in patients 1 and 2 have been studied previously; however, no mutations have been demonstrated. ${ }^{4}$

MONOCLONAL ANTIBODIES

The monoclonal antibodies used in the present study were $\mathrm{H} 11$ (anti- $\alpha 1$ ), H22 (anti- $\alpha 2$ ), H31 (anti- $\alpha 3$ ), H43 (anti- $\alpha 4$ ), H52 (anti- $\alpha 5$ ), and H63 (anti- $\alpha 6$ ). ${ }^{11}$ They were prepared against synthetic peptides of non-consensus regions of human $\alpha(I V)$ non-collagenous domains. Specificity was confirmed using western blotting and epitope mapping.

\section{IMMUNOHISTOCHEMISTRY}

Paraffin wax embedded renal sections were used for the analysis, although monoclonal antibodies are not very reactive with such sections; antigen retrieval was used to make the method more sensitive. The antigen retrieval procedure was as follows: the sections were heated in a small autoclave (KT2322, ALP Company Ltd, Tokyo, Japan) at $110-127^{\circ} \mathrm{C}$ for six minutes while they were immersed in $0.2 \mathrm{M}$ $\mathrm{HCl}$ ( $\mathrm{pH} \mathrm{0.9).}{ }^{12}$ Subsequently, the monoclonal antibodies were applied to the sections. Bound monoclonal antibodies were detected using the LSAB kit (Dako, California, USA). To avoid technical errors, immunostaining was performed at least twice on sections heated at more than two different temperatures.

\section{CONTROLS}

Two renal specimens from patients without Alport's syndrome or Epstein's syndrome were stained according to the same method to act as positive controls. The recovery of antigenicity was poor in sections stored for extended periods. Renal specimens from patients 1, 2, and 3 were prepared between 1989 and 1994 . Controls 1 and 2 were prepared in 1994 and 1989 , respectively.

\section{Results}

CONTROLS

A combination of chain specific monoclonal antibodies and antigen retrieval revealed well demonstrated $\alpha 1$ (IV) through to $\alpha 6$ (IV) chains in the renal sections of controls 1 and 2. $\alpha$ (IV) and $\alpha 2$ (IV) chains were expressed ubiquitously in all basement membranes and mesangial matrices, whereas the other $\alpha(I V)$ chains showed a more limited distribution. $\alpha 3$ (IV) and $\alpha 4$ (IV) chains were coexpressed in the glomerular basement membrane and in a portion of tubular basement membrane. They also appeared in Bowman's capsular basement membrane at a low intensity. The $\alpha 5$ (IV) chain was detected in the glomerular basement membrane, together with the $\alpha 3$ (IV) and $\alpha 4$ (IV) chains. $\alpha 5$ (IV) and $\alpha 6$ (IV) chains were detected in Bowman's capsular basement membrane and a portion of the tubular basement membrane. 

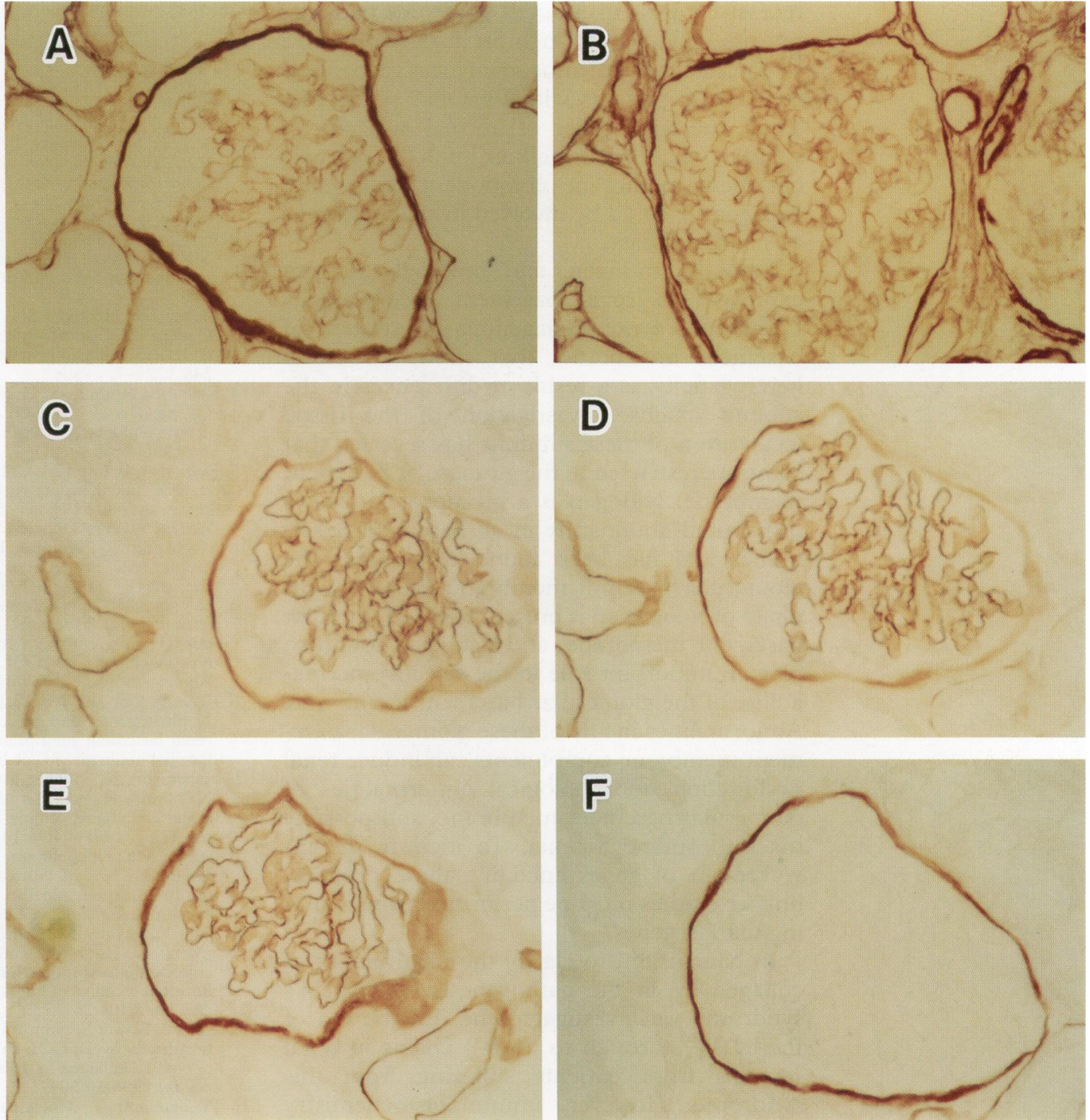

Figure 2 Distribution of a (IV) chains in a renal sample from patient 1. (A) a1 (IV); (B) a2 (IV);(C) a3(IV); (D) a $4(I V)$; (E) a.5(IV); (F) a6(IV). All a (IV) chains are distributed in a normal pattern and show normal antigenicity. a1(IV) and a2(IV) chains are found in all renal basement membranes and in mesangial matrix. $03(I V)$ and a $4(I V)$ chains are detected in glomerular basement membrane, a portion of tubular basement membrane, and half of the Bowman's capsular basement membrane. a5(IV) and a6(IV) chains are detected throughout Bowman's capsule basement membrane and in a portion of tubular basement membrane. The a $5(I V)$ chain, but not the a6(IV) chain, is also detected in the glomerular basement membrane.

\section{PATIENTS}

All three patients demonstrated significant positive staining for all $\alpha$ (IV) chains in the renal basement membranes. Figure 2 shows the results obtained from patient 1. Antigen retrieval demonstrated positive staining of $\alpha 1$ (IV) to $\alpha 6$ (IV) chains, with the same intensity and pattern of staining as seen in the controls. No significant abnormalities were found in the Epstein's syndrome cases.

\section{Discussion}

Alport's syndrome is classified into several subtypes according to its inheritance forms and accompanying symptoms. An X linked dominant form of Alport's syndrome has been found to result from mutations in the COL4A5 gene, ${ }^{3-5}$ and an autosomal recessive form has been shown to result from mutations in the COL4A3 and COL4A4 genes. ${ }^{67}$ Immunohistochemical studies of $\alpha$ (IV) chains has revealed characteristic abnormalities in $\alpha$ (IV) chain distribution corresponding to the causative mutations in the collagen IV genes. Immunohisto- chemical abnormalities of the $\alpha$ (IV) chains in $\mathrm{X}$ linked dominant Alport's syndrome have been well documented. ${ }^{8911} 12$ In male patients, deletion mutations in COL4A5 result in the complete absence of $\alpha 3$ (IV) to $\alpha 6$ (IV) chains in renal basement membranes, ${ }^{8} 91112$ whereas missense mutations (glycine substitution) result in a reduction in the antigenicity of these chains. ${ }^{12}$ In female patients, discontinuity of the antigenicity of $\alpha 3$ (IV) through to $\alpha 5$ (IV) chains has been described. ${ }^{9}$ In an autosomal recessive form of Alport's syndrome, immunohistochemistry has revealed a complete absence of $\alpha 3$ (IV) and $\alpha 4$ (IV) chains associated with the partial absence of $\alpha 5$ (IV) chains in the glomerular basement membrane. ${ }^{10}$ In the present study, Epstein's patients demonstrated normal antigenicity in all $\alpha$ (IV) chains in the renal basement membranes, raising the possibility that genes other than those coding for collagen IV are involved in the pathogenesis of the disease.

Normal antigenicity of collagen IV has also been reported in $\mathrm{X}$ linked ${ }^{12}$ and autosomal 
recessive types ${ }^{10}$ of Alport's syndrome patients, but the incidence of such findings is very low. In contrast, normal antigenicity was recognised in all three patients with Epstein's syndrome. Thus, the appearance of normal antigenicity suggests that abnormalities of a glomerular basement membrane component other than collagen IV could be involved in the pathogenesis of the disease.

Laminin, nidogen, and proteoglycans are expressed in renal basement membranes. ${ }^{13}$ Laminin is a non-collagenous constituent of basement membranes that shows structural heterogeneity as a result of differences in the subunit chains. Investigation of the tissue localisation of these subunits has revealed that some are restricted to the glomerular basement membrane. Nidogen is a glycoprotein that is distributed abundantly in all basement membranes and has been found throughout the glomerular basemnt membrane. Heparan sulphate proteoglycan is present in the glomerular basement membrane, and has been found to play an important role in the selective permeability of the glomerular basement membrane. Abnormalities in any of these glomerular basement membrane components can induce renal dysfunction, morphological abnormalities of glomerular basement membrane, and possibly macrothrombocytopenia. To identify the involvement of genes encoding these proteins, linkage studies must be performed using flanking marker genes.

In conclusion, to clarify the involvement of collagen IV in the pathogenesis of Epstein's syndrome we investigated the distribution of the $\alpha 1$ (IV) through to $\alpha 6$ (IV) chains in renal sections from patients suffering from this syndrome. However, immunohistochemistry revealed no abnormalities associated with collagen IV molecules.
This work was supported in part by a Grant in Aid for general Scientific Research (C) from the Japanese Ministry of Education, Science and Culture (07671270 and 08671312) and by a Research Project Grant from Kawasaki Medical School by a Research Project Grant from

1 Gregory MC, Terreros DA, Barker DF, Fain PN, Denison JC, Atkin CL. Alport syndrome-clinical phenotypes, incidence, and pathology. In: Tryggvason K, ed. Molecular, pathology and genetics of Alport syndrome. Contributions for nephrology, Basel: Karger, 1996;117:1-28.

2 Epstein's CJ, Sahud MA, Piel CF, Goodman JR, Bernfield $\mathrm{MR}$, Kushner JH, et al. Hereditary macrothrombocytopathia, nephritis and deafness. Am $\mathcal{F}$ Med 1972;52:299 310.

3 Barker DF, Hostikka SL, Zhou J, Chow LT, Oliphant AR, Gerken SC, et al. Identification of mutations in the COL4A5 collagen gene in Alport syndrome. Science 1990; 248:1224-7.

4 Kawai S, Nomura S, Harano T, Harano K, Fukushima T, Osawa G, et al. The COL4A5 gene in Japanese Alport syndrome patients: spectrum of mutations of all exons. Kidney Int 1996;49:814-22.

5 Renieri A, Bruttini M, Galli L, Zanelli P, Neri T, Rossetti S, et al. X-linked Alport syndrome: an SSCP-based mutation survey over all 51 exons of the COL4A 5 gene. $A m$ f Human Genet 1996;58:1192-204.

6 Mochizuki T, Lemmink $\mathrm{HH}$, Mariyama M, Antignac C, Gubler MC, Pirson Y, et al. Identification of mutations in the $\alpha 3$ (IV) and $\alpha 4$ (IV) collagen genes in autosomal the $\alpha 3(\mathrm{IV})$ and $\alpha 4$ (IV) collagen genes in autos

7 Lemmink HH, Mochizuki T, van den Heuvel LPWJ, Schroder CH, Barrientos A, Monnes LAH, et al. Mutation in the type IV collagen $\alpha 3$ (COL4A3) gene in autosomal recessive Alport syndrome. Hum Mol Genet 1994;3:1269-73.

8 Kashtan CE, Fish AF, Kleppel M, Yoshioka K, Michael AF. Nephritogenic antigen determinants in epidermal and renal basement membranes of kindreds with Alport-type familial nephritis. F Clin Invest 1986;78:1035-44.

9 Nakanishi K, Yoshikawa N, Iijima K, Kitagawa K, Nakamura $\mathrm{H}$, Ito $\mathrm{H}$, et al. Immunohistochemical study of alpha 1-5 chains of type IV collagen in hereditary nephritis. Kidney Int 1994;46:1413-21.

10 Gubler MC, Knebelmann B, Beziau A, Broyer M, Pirson Y Haddoum F, et al. Autosomal recessive Alport syndrome: immunohistochemical study of type IV collagen chain distribution. Kidney Int 1995;47:1 142-7.

11 Sado Y, Kagawa M, Kishiro Y, Sugihara K, Naito I, Seyer $\mathrm{JM}$, et al. Establishment by rat lymph node method of epitope-defined monoclonal antibodies recognizing the six different $\alpha$ chains of human type IV collagen. Histochem Cell Biol 1995;104:267-75.

12 Naito I, Kawai S, Nomura S, Sado Y, Osawa G, et al. Relationship between COL4A5 gene mutation and distribution tionship between COL4A5 gene mutation and distribution of type IV collagen in ma
ney Int 1996;50:304-11.

13 Pihlajaniemi T. Molecular properties of the glomerular basement membrane. In: Tryggvason K, ed. Molecular pathology and genetics of Alport syndrome. Contributions for nephrology. Basel: Karger, 1996;117:46--79. 